\title{
Effects of Continuum Breakdown on Hypersonic Aerothermodynamics
}

\author{
Andrew J. Lofthouse ${ }^{*}$ and Iain D. Boyd ${ }^{\dagger}$ \\ Department of Aerospace Engineering \\ University of Michigan, Ann Arbor, MI, 48109-2140 \\ Michael J. Wright \\ Reacting Flow Environments Branch \\ NASA Ames Research Center, Moffett Field, CA, 94035
}

\begin{abstract}
Hypersonic vehicles experience different flow regimes during flight due to changes in atmospheric density. Hybrid Computational Fluid Dynamics (CFD) and direct simulation Monte Carlo (DSMC) methods are being developed to simulate the flow in different hypersonic regimes. These methods use a breakdown parameter to determine regions of the flow where the CFD physics are no longer valid. The current study investigates the effect of continuum breakdown on surface aerothermodynamic properties, such as pressure, shear stress and heat transfer rate, of a cylinder in a Mach 10 flow of argon gas for several different flow regimes, from the continuum to a rarefied gas. CFD and DSMC solutions are obtained at each flow condition. Total drag predictions are identical for a continuum flow while differing by more than $\mathbf{2 6 \%}$ for a rarefied flow. Peak heat transfer rate differences range from less than $1 \%$ for a continuum flow to almost $32 \%$ for a rarefied flow. Drag depends primarily on continuum breakdown in the wake, while heat transfer rate appears to depend primarily on continuum breakdown in the shock and differences in thermal boundary layer thickness.
\end{abstract}

\section{Nomenclature}

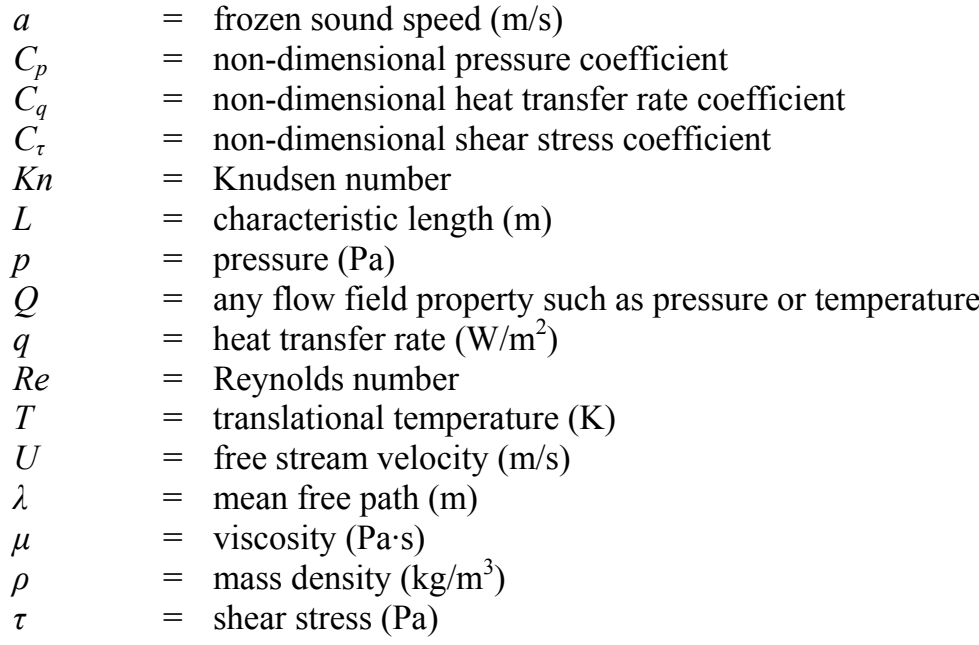

\footnotetext{
* Graduate Student, Member AIAA.

${ }^{\dagger}$ Professor, Associate Fellow AIAA.

* Senior Research Scientist, Senior Member AIAA.

"The views expressed in this paper are those of the author and do not reflect the official policy or position of the United States Air Force, Department of Defense, or the U.S. Government" 


\section{Introduction}

A hypersonic vehicle such as a planetary entry capsule or a reusable launch vehicle, will experience vastly different flow regimes during the course of its flight trajectory because the earth's atmosphere varies in density as a function of altitude. Reproduction of these varied flow conditions in ground-based laboratory facilities is both expensive and technically challenging. Hence, there is an extremely important role for computational models in the development of hypersonic vehicles.

The physical processes in gas dynamics can be conveniently characterized using the Reynolds number $R e$ and the Knudsen number $K n$ defined as follows:

$$
\operatorname{Re}=\frac{\rho U L}{\mu} \quad K n=\frac{\lambda}{L} \propto \frac{1}{\rho L}
$$

where $\rho$ is density, $U$ is velocity, $L$ is a characteristic length of the flow, $\mu$ is viscosity, and $\lambda$ is the mean free path. At low altitudes, the atmospheric density is relatively high, and flows around hypersonic vehicles should be simulated using traditional Computational Fluid Dynamics (CFD) by solving either the Euler or preferably the Navier-Stokes (NS) equations. ${ }^{1}$ This is the continuum regime characterized by very large Reynolds numbers and very low Knudsen numbers. At very high altitudes, at the edge of the atmosphere, the density is very low such that there are very few collisions between the molecules and atoms in the flow around the vehicle. This is the rarefied flow regime and can be computed using the direct simulation Monte Carlo (DSMC) method. ${ }^{2}$ Generally speaking, CFD methods for solving the NS equations are about an order of magnitude faster than the DSMC method. However, the lack of collisions makes the physics of the NS equations invalid. The rarefied regime is characterized by a small Reynolds number and a large Knudsen number. Note that in high-Re continuum regimes, locally a flow may behave like a low-Re/high-Kn rarefied flow if the local characteristic length scale is very small. This can be an important consideration as very sharp structures are being considered as an effective way to decrease drag on wing leading edges and for effective engine inlet control. Alternatively, on a blunt body, a high-density fore-body flow can create a rarefied flow in the wake of the vehicle. In principle, the DSMC method can be applied to any dilute gas flow, but becomes prohibitively expensive for Knudsen numbers less than 0.001. Thus, either CFD or DSMC on its own fails to provide a comprehensive computational modeling capability across all flow regimes encountered by a hypersonic vehicle.

A natural solution to this problem is to develop a hybrid simulation technique that employs a CFD method for as much of the flow field as possible (due to its superior numerical performance) and a DSMC method in regions of the flow where the physics description provided by the CFD method is inadequate. Development of such hybrid methods is an area of active research. ${ }^{3,4}$ For near continuum flows, the CFD method would be used in the majority of the flow field while DSMC would be used in the shock and the wake regions.

A key part of any hybrid method is a reliable means to determine when to switch between CFD and DSMC within the computational domain. In general, such approaches rely on predicting incipient failure of the continuum (CFD) equations, using a so-called continuum breakdown parameter. For example, Boyd, et al, ${ }^{5}$ carried out extensive numerical investigation of one-dimensional normal shock waves and two-dimensional bow shocks comparing DSMC and CFD results to determine an appropriate breakdown parameter. They proposed the use of the gradient-length local (GLL) Knudsen number

$$
K n_{G L L}=\frac{\lambda}{Q}\left|\frac{d Q}{d l}\right|
$$

where $l$ is some distance between two points in the flow field, and $Q$ is some quantity of interest such as density, pressure or temperature, provides an effective indication of continuum breakdown for hypersonic compressed flows. It is generally assumed that continuum breakdown occurs whenever $\mathrm{Kn}_{\mathrm{GLL}}$ is greater than 0.05 .

For design purposes, it is equally important to be able to characterize the effect of a given level of continuum breakdown on design variables of interest. For hypersonic cruise or planetary entry vehicles, the primary design variables are all surface quantities: heat flux, pressure and shear stress. These variables govern not only the aerodynamic performance of the vehicle, but also determine the selection and sizing of the thermal protection system (TPS), which protects the vehicle from the entry environment. However, a quantitative link between a given level of continuum-breakdown and the accuracy of predicted surface quantities using CFD has not been presented in 
prior studies in the literature. The goal of the present study is therefore to investigate this fundamental issue. Specifically, how are the critical hypersonic vehicle design surface properties of pressure, shear stress and heat transfer rate affected by failure of the continuum approach in certain regions of the flow field. For example, in hypersonic flow, the first place where continuum breakdown is observed is within the shock wave itself. It is well known that traditional continuum CFD cannot accurately predict shock structure correctly under any circumstances. ${ }^{6}$ It is not clear, however, whether local breakdown within the shock has a tangible impact on the rest of the flow field and the resulting surface properties.

This paper will first briefly describe the simulation procedures used, including some background of the computational models. It will then discuss general flow field and surface property results predicted by the models in several different flow regimes, from the continuum to a rarefied gas. A short comparison with analytical freemolecular results will also be presented. Finally, some conclusions and future work recommendations are presented.

\section{Background and Simulation Procedure}

This investigation considers a Mach 10 hypersonic flow of argon (free stream temperature and velocity of $2634.1 \mathrm{~m} / \mathrm{s}$ and $200 \mathrm{~K})$ over a two-dimensional, 12-inch diameter cylinder that has a fixed surface temperature of $500 \mathrm{~K}$, as shown in Figure 1. The free stream density of the flow is varied such that several different regimes are considered, from the continuum through the transitional to the rarefied regime, as shown in Table 1. Knudsen numbers are calculated based on the cylinder diameter. Surface and flow field properties for this flow are presented from two different computational approaches.

First, CFD results are obtained through solution of the Navier-Stokes equations. The CFD results are obtained using the Data-Parallel Line Relaxation (DPLR) code, ${ }^{7}$ developed at NASA Ames Research Center for the simulation of hypersonic reacting flow-fields. DPLR includes generalized models for nonequilibrium chemical kinetics, energy relaxation and surface reactions, and has been employed for the design and analysis of many Earth and planetary entry vehicles. ${ }^{8,9}$ All DPLR solutions are generated assuming an isothermal wall at $500 \mathrm{~K}$. A no-slip velocity and temperature boundary condition is enforced. In each case, a grid size of $120 \times 120$ cells is employed, and the wall spacing is chosen to ensure that the cell Reynolds number $\left(\operatorname{Re}_{\mathrm{c}}=\rho \mathrm{a} \Delta \eta / \mu\right)$ at the wall is less than one everywhere on the cylinder.

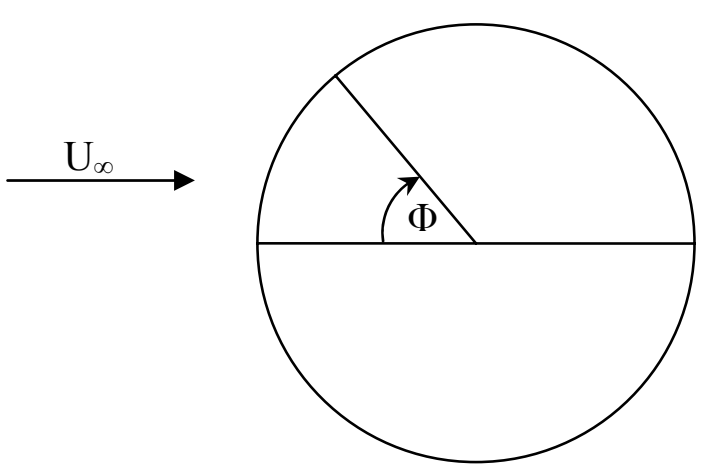

Figure 1. Geometry definition.

Table 1. Flow regimes considered.

\begin{tabular}{|l|l|l|}
\hline \multicolumn{1}{|c|}{ Kn } & $\begin{array}{c}\text { Mass Density, } \\
\mathrm{kg} / \mathrm{m}^{3}\end{array}$ & $\begin{array}{c}\text { Number Density, } \\
\text { particles } / \mathrm{m}^{3}\end{array}$ \\
\hline \hline 0.002 & $1.408 \times 10^{-4}$ & $2.124 \times 10^{21}$ \\
\hline 0.01 & $2.818 \times 10^{-5}$ & $4.247 \times 10^{20}$ \\
\hline 0.05 & $5.636 \times 10^{-6}$ & $8.494 \times 10^{19}$ \\
\hline 0.25 & $1.127 \times 10^{-6}$ & $1.699 \times 10^{19}$ \\
\hline
\end{tabular}

Second, DSMC results are provided from the MONACO $\operatorname{code}^{10}$ for the same flow conditions. MONACO is a general 2D/3D, parallel, unstructured mesh DSMC code that has been applied to many hypersonic, rarefied flows. ${ }^{11,12}$ All MONACO solutions are generated using a fixed wall temperature at $500 \mathrm{~K}$. Bird's variable hard sphere model is used. ${ }^{2}$ In general, the mesh used for the final solution for each case is adapted from previous solutions such that each cell size is on the order of a mean free path. The exception is the $\mathrm{Kn}=0.002$ case, where the cell size is approximately four times the mean free path and the subcell method is used to select particles for collisions. ${ }^{2}$

The viscosity and thermal conductivity of pure argon in the CFD code are computed from collision integrals presented by Murphy and Arundell, ${ }^{13}$ as recommended in the recent review of Wright et al. ${ }^{14}$ The variable hard sphere parameters used in the DSMC computations are selected to be consistent with this CFD viscosity model.

The computational cost of each type of simulation is provided in Table 2. In each case, the CFD results take one hour or less of total CPU time to complete. The DSMC results, on the other hand, take several hundreds, even thousands of hours in total CPU time. (It should be noted here that the $\mathrm{Kn}=0.25$ case could have been completed with far fewer particles.) The accuracy of the DSMC method in regions of high non-equilibrium motivates the development of hybrid CFD/DSMC methods, where the computational cost would be significantly reduced. 
Table 2. DSMC and CFD computational costs.

\begin{tabular}{|c|c|c|c|c|c|c|c|c|c|}
\hline \multirow{2}{*}{ Kn } & \multicolumn{4}{|c|}{ DSMC } & \multicolumn{4}{c|}{ CFD } \\
\cline { 2 - 11 } & Cells & Particles & Iterations & CPUs & $\begin{array}{c}\text { Total CPU } \\
\text { Time* (hrs) }\end{array}$ & Cells & Iterations & CPUs & $\begin{array}{c}\text { Total CPU } \\
\text { Time* (hrs) }\end{array}$ \\
\hline 0.002 & 58,078 & $96.0 \times 10^{6}$ & $8.2 \times 10^{5}$ & 64 & $20,185.6$ & 14,400 & 1,500 & 10 & 0.38 \\
\hline 0.01 & 34,770 & $26.8 \times 10^{6}$ & $4.3 \times 10^{5}$ & 32 & $1,827.2$ & 14,400 & 3,000 & 10 & 0.78 \\
\hline 0.05 & 9,661 & $7.1 \times 10^{6}$ & $4.3 \times 10^{5}$ & 32 & 499.2 & 14,400 & 4,000 & 10 & 1.03 \\
\hline 0.25 & 12,447 & $16.5 \times 10^{6}$ & $2.9 \times 10^{5}$ & 32 & $1,030.4$ & 14,400 & 4,000 & 10 & 1.03 \\
\hline
\end{tabular}

* Total CPU Time is wall time multiplied by number of CPUs used

\section{Results}

This investigation seeks to correlate changes in continuum breakdown of the hypersonic flow around the cylinder with changes in surface properties. The overall flow features, such as temperature and density fields, are compared, as well as the surface distributions of pressure, shear stress and heat transfer. A comparison of the maximum values of the breakdown parameter for each case is shown in Table 3. The breakdown parameter is calculated using both the CFD and the DSMC solutions using Equation (2) with $l$ being the length of a streamline in a computational cell. The CFD solution is computed using two zones, one for the fore-body area and one for the wake region (the separation point is at about $\Phi=120^{\circ}$; see Figure 1); Table 3 shows the maximum value for the breakdown parameter in each zone. Recalling that $\mathrm{Kn}_{\mathrm{GLL}}>0.05$ indicates continuum breakdown, it is interesting that all flows investigated here experience breakdown.

Note that the extremely large breakdown parameter computed from the CFD solution for $\mathrm{Kn}=0.25$ is not realistic. The CFD solution predicts a density value on the lee side of the cylinder (at $\Phi=180^{\circ}$ ) on the order of $1 \times 10^{-13} \mathrm{~kg} / \mathrm{m}^{3}$. Since the mean free path is proportional to the inverse of the density, this causes the mean free path to be extremely large, and hence the breakdown parameter is also extremely large. The CFD solution is not expected to be accurate in such a rarefied flow, and so these extremely high breakdown parameter values are not expected to be accurate either. Nevertheless, in a hybrid CFD-DSMC code using $\mathrm{Kn}_{\mathrm{GLL}}$ as a breakdown parameter, this condition would certainly flag the solver that the CFD solution is not valid in this specific region. The DSMC solutions are expected to be more realistic at this condition.

Figures 2-5 illustrate the increase in continuum breakdown, as quantified by $\mathrm{Kn}_{\mathrm{GLL}}$ computed from the CFD results, as the flow becomes more rarefied. In general, the flow experiences continuum breakdown in two areas; across the bow shock and in the wake region. The flow in the shock region experiences very steep gradients in flow properties, while the wake region is more rarefied, thus leading to the breakdown of the continuum hypothesis.

The maximum breakdown in the fore-body region (due to the shock) increases as the flow becomes more rarefied, although the change is not very large. In contrast, the breakdown in the wake region increases as the density decreases. This increased breakdown in the wake seems to have a large effect on the shear stress.

Table 3. Maximum gradient-length local Knudsen numbers, based on flow field properties according to Eq. (2), computed from CFD and DSMC solutions.

\begin{tabular}{|l|l|l|l|l|l|l|l|l|l|}
\hline \multirow{3}{*}{ Kn } & \multicolumn{4}{|c|}{ Density } & \multicolumn{3}{c|}{ Pressure } & \multicolumn{3}{c|}{ Temperature } \\
\cline { 2 - 6 } & \multicolumn{3}{|c|}{ CFD } & \multirow{2}{*}{ DSMC } & \multicolumn{3}{c|}{ CFD } & \multirow{2}{*}{ DSMC } & \multicolumn{3}{c|}{ CFD } & \multirow{2}{*}{ DSMC } \\
\cline { 2 - 7 } & Forebody & Wake & & Forebody & Wake & & Forebody & Wake & \\
\hline 0.002 & 0.15 & 0.18 & 0.63 & 0.62 & 0.22 & 0.78 & 0.46 & 0.18 & 0.62 \\
\hline 0.01 & 0.36 & 0.57 & 0.21 & 1.17 & 0.30 & 0.77 & 1.12 & 0.59 & 0.55 \\
\hline 0.05 & 0.97 & 35.54 & 0.95 & 1.62 & 57.2 & 1.90 & 1.57 & 31.45 & 1.17 \\
\hline 0.25 & 6.32 & $1.35 \times 10^{7}$ & 38.19 & 2.74 & $1.97 \times 10^{7}$ & 53.40 & 5.91 & $1.01 \times 10^{7}$ & 19.30 \\
\hline
\end{tabular}


Table 4. Total drag.

\begin{tabular}{|l|l|l|l|}
\hline \multirow{2}{*}{ Kn } & \multicolumn{2}{|c|}{ Drag/Length, N/m } & \multirow{2}{*}{ Difference } \\
\cline { 2 - 3 } & \multicolumn{1}{|c|}{ DSMC } & \multicolumn{1}{|c|}{ CFD } & \\
\hline \hline 0.002 & 189 & 189 & $0.0 \%$ \\
\hline 0.01 & 40.0 & 40.2 & $0.5 \%$ \\
\hline 0.05 & 8.91 & 9.45 & $6.0 \%$ \\
\hline 0.25 & 2.08 & 2.63 & $26.2 \%$ \\
\hline
\end{tabular}

Table 5. Peak heat transfer rate.

\begin{tabular}{|l|l|l|l|}
\hline \multirow{2}{*}{ Kn } & \multicolumn{2}{|c|}{ Peak Heating, W/m ${ }^{2}$} & \multirow{2}{*}{ Difference } \\
\cline { 2 - 4 } & \multicolumn{1}{|c|}{ DSMC } & \multicolumn{1}{|c|}{ CFD } & \\
\hline \hline 0.002 & 92,522 & 93,104 & $0.6 \%$ \\
\hline 0.01 & 39,319 & 40,884 & $4.0 \%$ \\
\hline 0.05 & 16,164 & 18,191 & $12.5 \%$ \\
\hline 0.25 & 5,984 & 7,889 & $31.8 \%$ \\
\hline
\end{tabular}

Tables 4 and 5 compare the total drag and the peak heat transfer rates predicted by both computational methods.

In the results that follow, the surface properties are presented in terms of non-dimensionalized coefficients with the following definitions:

$$
C_{p}=\frac{p}{\rho_{\infty} U_{\infty}^{2}} \quad C_{\tau}=\frac{\tau}{\rho_{\infty} U_{\infty}^{2}} \quad C_{q}=\frac{q}{\rho_{\infty} U_{\infty}^{3}}
$$

where $p$ is the pressure, $\tau$ is the shear stress, $q$ is the heat transfer rate, $\rho_{\infty}$ is the free stream density and $U_{\infty}$ is the free stream velocity. The surface properties in each case are plotted as a function of the angle around the cylinder, with the stagnation point being located at an angle of zero (Figure 1).

\section{A. $\mathrm{Kn}=\mathbf{0 . 0 0 2}$}

The current investigation only considers the flow over the fore-body of the cylinder for this case due to the significant computational expense of the DSMC method.

At a Knudsen number of 0.002 , the flow is well within the continuum regime. Nevertheless, there is still evidence of continuum breakdown in the shock (Figure 2), although it is only a small amount. This level of breakdown is not expected to significantly affect the surface properties. Interestingly, DSMC predicts a larger degree of breakdown than does CFD (Table 3).

The temperature and density fields predicted by CFD and DSMC are in excellent agreement, as expected (Figures 6 and 7). The surface pressure (Figure 8) shows very good agreement, until the point at which the DSMC domain is truncated, where the surface pressure drops. The DSMC shear stress (Figure 9) contains a large amount of statistical noise, indicating that more sampling iterations are required. Nevertheless, the agreement is good up to the point of DSMC domain truncation, where there is a sharp increase in shear stress predicted by DSMC. The heat transfer rate (Figure 10) also shows fair agreement, but there is again some evidence of statistical noise in the DSMC results. Since the truncation of the domain introduces additional error in the surface properties at the edge of the domain, the drag value shown in Table 4 is the integrated drag up to a point at about $\Phi=86^{\circ}$. The total drag predicted by both methods is identical (to four significant digits) and peak heat transfer rates differ by less than $1 \%$. These results are well within the error range expected for a flow for which both methods are valid.

Further investigations are planned to simulate the entire flow field around the cylinder.

\section{B. $\mathrm{Kn}=\mathbf{0 . 0 1}$}

This Knudsen number is considered to be near the limit of the continuum regime. Here there is increased evidence of continuum breakdown (Figure 3) although the maximum value of the breakdown parameter predicted by CFD tends to be higher than that predicted by DSMC (Table 3). In this case, the shock shows more breakdown than the wake. However, comparison of general flow field features shows that the two numerical solutions (CFD and DSMC) do not differ by a large amount. The temperature field (Figure 11) is very similar, with a few exceptions in the shock structure and the wake, where the continuum hypothesis is expected to break down first. The shock standoff distance predicted by both methods is the same, as is the maximum temperature behind the shock. CFD shows a larger thermal boundary layer than DSMC. DSMC also predicts a thicker shock, as expected. The thicker shock does not seem to have an effect on the surface properties, but the smaller thermal boundary layer does seem to affect the heat transfer rate, as is shown below. Also, the minimum temperature in the wake predicted by CFD is less than 
that predicted by DSMC. The density field (Figure 12) is also very similar, especially in the fore-body region. The exception is the wake region, where CFD predicts an overall higher density than DSMC, as well as a pocket of higher density directly behind the cylinder than in the surrounding wake.

The amount of breakdown in the shock does not necessarily carry over to the surface properties. The surface pressure predicted by both methods is very nearly equal over the entire cylinder (Figure 13). The shear stress also shows very good comparison up to the peak value, but then DSMC predicts slightly less shear stress than CFD in the wake (Figure 14). The difference can be attributed to the more rarefied nature of the flow in this region predicted by DSMC. The total drag due to pressure and viscous effects predicted by CFD is within $0.5 \%$ of that predicted by DSMC (Table 4). The heat transfer rate shows more of a difference between CFD and DSMC along the entire surface (Figure 15). However, the difference in the present case is not very significant. The peak heating also differs by less than $4 \%$ (Table 5 ). These results indicate that the surface properties are not strongly affected by the continuum breakdown in the shock.

\section{C. $\mathrm{Kn}=\mathbf{0 . 0 5}$}

At a Knudsen number of 0.05 , the flow is generally considered to be outside the continuum regime. Thus, the CFD results are not expected to be entirely accurate. The flow demonstrates breakdown in a larger area of the flow, primarily in the wake (Figure 4), although the maximum values of the breakdown parameter computed from the CFD solution in the wake are much larger than that computed from the DSMC solution (Table 3). The differences between the CFD and DSMC temperature (Figure 16) and density fields (Figure 17) are more pronounced than the lower Knudsen number cases. The DSMC shock is much thicker than the CFD shock, although the shock stand-off distance and peak temperatures are still very nearly equal. The CFD results also show a larger thermal boundary layer than the DSMC solution. In the wake, DSMC predicts a lower density and a higher temperature than CFD.

The surface pressure predicted by both methods is still in excellent agreement (Figure 18). However, the shear stress is higher in the CFD results, especially in the wake (Figure 19). The point at which the CFD and DSMC shear stress results diverge is farther forward along the cylinder surface than at lower Knudsen number, and the DSMC peak shear stress is lower by about $12 \%$. The heat transfer rate differs by an almost uniform amount along the entire surface, and the difference is larger than at lower Knudsen numbers (Figure 20). The difference in total drag is within $6 \%$ (Table 4). On the other hand, the peak heat transfer rate differs by more than $12 \%$ (Table 5 ). Again, the pressure does not seem to be affected by continuum breakdown, while the shear stress seems to be affected more by the breakdown in the wake, and the heat transfer rate by the breakdown in the shock and the larger thermal boundary layer. The sensitivity of the shear stress and heat transfer to continuum breakdown is most likely due to the no-slip boundary condition imposed at the wall in the CFD method, which is invalid for higher Knudsen number flows.

\section{D. $\mathrm{Kn}=\mathbf{0 . 2 5}$}

At a Knudsen number of 0.25 , the flow is well within the rarefied regime, so large errors in flow properties are expected from the CFD method. There is more breakdown in the flow, in terms of the size of the breakdown region and the value of the breakdown parameter (Figure 5). Note that the CFD breakdown parameter in the wake is not expected to be accurate as mentioned above (Table 3). Nevertheless, the value of the DSMC breakdown parameters are also much larger than that for previous cases; hence the continuum hypothesis is definitely not valid in those regions. The temperature field (Figure 21) shows that the DSMC shock is much thicker, and the maximum temperature behind the shock predicted by DSMC is higher than that predicted by CFD (although the CFD heat transfer rate is still higher than the DSMC rate as discussed below). The thermal boundary layer is again larger in the CFD results than in the DSMC results. However, the shock standoff distance appears to be fairly similar. The CFD wake is predicted to be more rarefied, as seen in the density field (Figure 22).

The surface pressure predicted by both methods is no longer in agreement (Figure 23). At this Knudsen number, the shock has partially merged with the boundary layer. The DSMC pressure is less than the CFD pressure near the fore-body, but the agreement does improve in the wake. As seen in Figure 5, the breakdown in the shock region extends much closer to the surface of the cylinder and so it most likely affects the surface pressure. The shear stress shows the same general trend as in previous cases in that both methods agree near the stagnation region, but the results diverge as the flow accelerates around the cylinder (Figure 24). The DSMC shear stress is lower than the CFD shear stress, with the peak DSMC shear stress about 33\% lower. The heat transfer rate also follows trends similar to the previous cases in that the DSMC heat transfer rate is lower than the CFD rate along the entire surface (Figure 25). The total drag predicted by CFD is higher than the DSMC drag by more than $26 \%$, due to the much higher CFD shear stress (Table 4). The peak heat transfer rate also differs by nearly $32 \%$ (Table 5 ). 


\section{E. Free-Molecular Comparison $(\mathrm{Kn}=\infty)$}

As the Knudsen number increases, the computational results for surface pressure, shear stress and heat transfer should approach the analytical results in the free-molecular, or collisionless, limit. ${ }^{15}$ As a further comparison of the DSMC implementation, a case is run for $\mathrm{Kn}=100$, which is essentially collisionless. Figures $26-28$ compare the non-dimensional surface properties obtained through simulation with those obtained analytically for free-molecular flow. It is evident that as the Knudsen number increases, the DSMC results do indeed approach the analytic results. In fact, for $\mathrm{Kn}=100$ the DSMC results are nearly identical to the analytical results. Although it appears that the CFD results tend toward the free-molecular limit earlier than the DSMC results, this is not indicative of higher accuracy at the higher Knudsen numbers, but is a result of the no-slip boundary condition imposed at the wall.

\section{Conclusion}

Comparison of CFD and DSMC results for similar flow conditions showed that the surface properties of pressure, shear stress and heat transfer rates were very similar for the lower Knudsen number flows where the continuum hypothesis is valid, as expected, while they diverged for the higher Knudsen number results. The surface pressure was least affected by continuum breakdown, as quantified by the gradient-length local Knudsen number, among those properties investigated, and seemed to be affected only by breakdown in the shock region at the highest Knudsen number flow. The shear stress was most influenced by continuum breakdown and was affected primarily by continuum breakdown in the wake. This sensitivity is most likely due to the no-slip condition imposed at the wall by the CFD method, which is not valid for higher Knudsen number flows. The heat transfer rate was consistently different along the entire surface of the cylinder in all cases, although the difference increased with the more rarefied flows. This difference is most likely due to breakdown in the shock and differences in the thermal boundary layer thickness. In all cases, the surface properties predicted by DSMC tended to be lower than those predicted by CFD, which also suggests a velocity slip and temperature jump at the cylinder wall. As the Knudsen number increased, the difference in surface properties predicted by CFD and DSMC increased from less than 1\% at $\mathrm{Kn}=0.002$ to more than $30 \%$ at $\mathrm{Kn}=0.25$.

In all cases, the CFD method was more conservative than the DSMC method, predicting higher drag and peak heat transfer rate. In the design of planetary entry vehicles, a conservative prediction of heat transfer may not be completely undesirable in that the thermal protection shield will be designed to withstand higher temperatures than is completely necessary, but would not adversely impact vehicle performance. However, accurate prediction of drag is important for complete understanding of flight vehicle dynamics and accurate prediction of the vehicle's trajectory.

\section{Future Work}

The results presented here are not comprehensive. Future investigations will explore the effect of continuum breakdown on the surface properties for an increased envelope of flow conditions. For example, flows at higher or lower Mach numbers could be investigated to determine any dependence of the correlation on flow speed. Agreement between the CFD and DSMC results should be improved by implementing a finite-slip and temperature jump boundary conditions at the wall for the CFD simulations. Modifications to the DPLR code are being performed to implement this feature and future simulations will be compared with DSMC results. Additionally, the results presented here are two-dimensional only; three-dimensional affects will be investigated by considering the flow around an axisymmetric sphere. Finally, the investigation considered only the flow of argon around the cylinder; future work will consider gases of more complex molecules with internal energy and chemical reactions.

\section{Acknowledgments}

The first author gratefully acknowledges the support of the Air Force Institute of Technology. This work is also sponsored in part by the Space Vehicle Technology Institute, under NASA grant NCC3-989 with joint sponsorship from the Department of Defense, and by the Air Force Office of Scientific Research, through grant FA9550-05-10115. The generous use of NASA high performance computing resources was indispensable to this investigation and is greatly appreciated. 


\section{References}

${ }^{1}$ Candler, G.V., Nompelis, I., and Druguet, M.C., "Navier-Stokes Predictions of Hypersonic Double-Cone and Cylinder-Flare Flow Field," AIAA Paper 2001-1024, January 2001.

${ }^{2}$ Bird, G.A., Molecular Gas Dynamics and the Direct Simulation of Gas Flows, Oxford University Press, Oxford, 1994.

${ }^{3}$ Wang, W.-L., Sun, Q., and Boyd, I.D., "Assessment of a Hybrid Continuum/Particle Approach for Hypersonic Flows," Proceedings of the 23rd International Symposium on Rarefied Gas Dynamics, Whistler, Canada, July 2002.

${ }^{4}$ Schwartzentruber, T.E. and Boyd, "Detailed Analysis of a Hybrid CFD-DSMC Method for Hypersonic Non-Equilibrium Flows," AIAA Paper 2005-4829, June 2005.

${ }^{5}$ Boyd, I. D., Chen, G., and Candler, G. V., "Predicting Failure of the Continuum Fluid Equations in Transitional Hypersonic Flows," Physics of Fluids, Vol. 7, 1995, pp. 210-219.

${ }^{6}$ Candler, G.V., S. Nijhawan, D. Bose, and I.D. Boyd, “A Multiple Temperature Gas Dynamics Model," Physics of Fluids, Vol. 6, No. 11, pp. 3776-3786, Nov. 1994.

${ }^{7}$ Wright, M.J., Candler, G.V., and Bose, D., "Data-Parallel Line Relaxation Method for the Navier-Stokes Equations," AIAA Journal, Vol. 36, No. 9, 1998, pp. 1603-1609.

${ }^{8}$ Wright, M.J., Loomis, M., and Papadopoulos, P., “Aerothermal Analysis of the Project Fire II Afterbody Flow," Journal of Thermophysics and Heat Transfer, Vol. 17, No. 2, 2003, pp. 240-249.

${ }^{9}$ Wright, M.J., Bose, D., and Olejniczak, J., "The Impact of Flowfield-Radiation Coupling on Aeroheating for Titan Aerocapture," Journal of Thermophysics and Heat Transfer, Vol. 19, No. 1, 2005, pp. 17-27.

${ }^{10}$ Dietrich, S., and Boyd, I.D., "Scalar and Parallel Optimized Implementation of the Direct Simulation Monte Carlo Method," Journal of Computational Physics, 126, 1996, pp. 328-342.

${ }^{11}$ Boyd, I.D. and Padilla, J.F., "Simulation of Sharp Leading Edge Aerothermodynamics," AIAA Paper 2003-7062, December 2003.

${ }^{12}$ Sun, Q., Cai, C., Boyd, I.D., Clemmons, J.H. and Hecht, J.H., "Computational Analysis of High-Altitude Ionization Gauge Flight Measurements," Journal of Spacecraft and Rockets, Vol. 42, 2005.

${ }^{13}$ Murphy, A. B., and Arundell, C. J., "Transport Coefficients of Argon, Nitrogen, Oxygen, Argon-Nitrogen and ArgonOxygen Plasmas," Plasma Chemistry and Plasma Processing, Vol. 14, No. 4, 1994, pp. 451-490.

${ }^{14}$ Wright, M.J., Bose, D., Palmer, G.E., and Levin, E., "Recommended Collision Integrals for Transport Property Computations I: Air Species," AIAA Journal, Vol. 43, No. 12, 2005, pp. 2558-2564.

${ }^{15}$ Gombosi, T., Gaskinetic Theory, Cambridge University Press, 1994. 


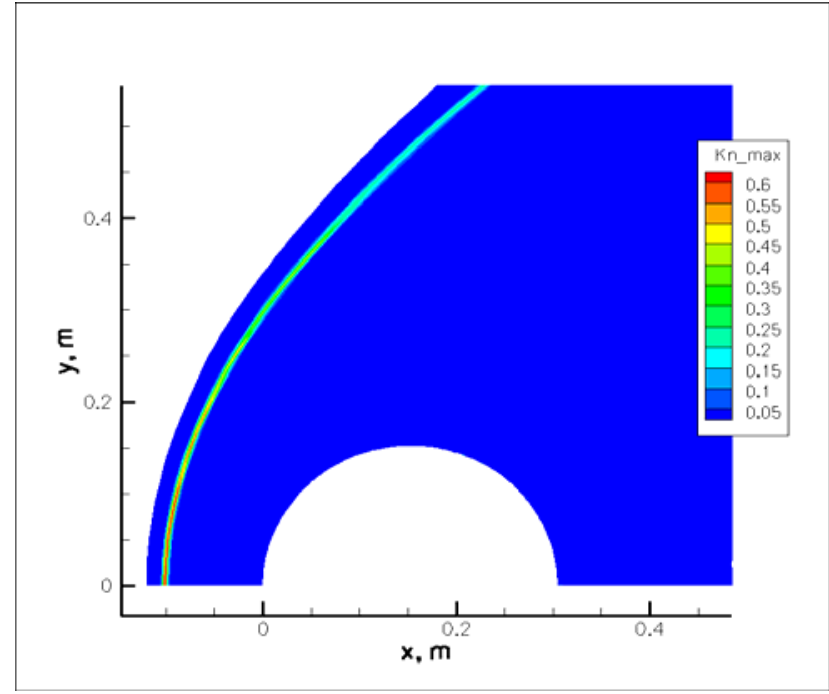

Figure 2. $\mathrm{Kn}=0.002$ maximum gradient length local Knudsen number.

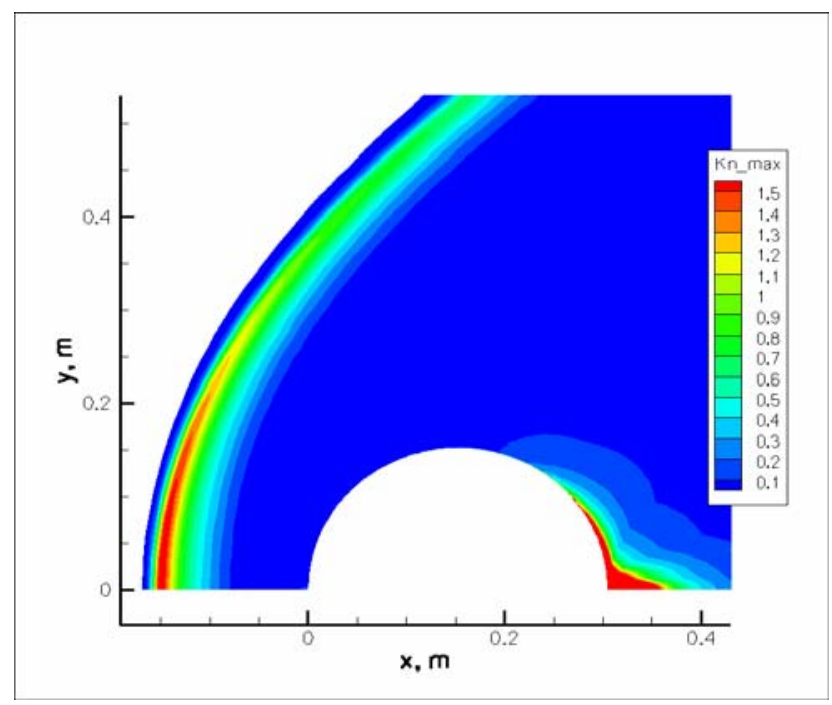

Figure 4. $\mathrm{Kn}=0.05$ maximum gradient length local Knudsen number.

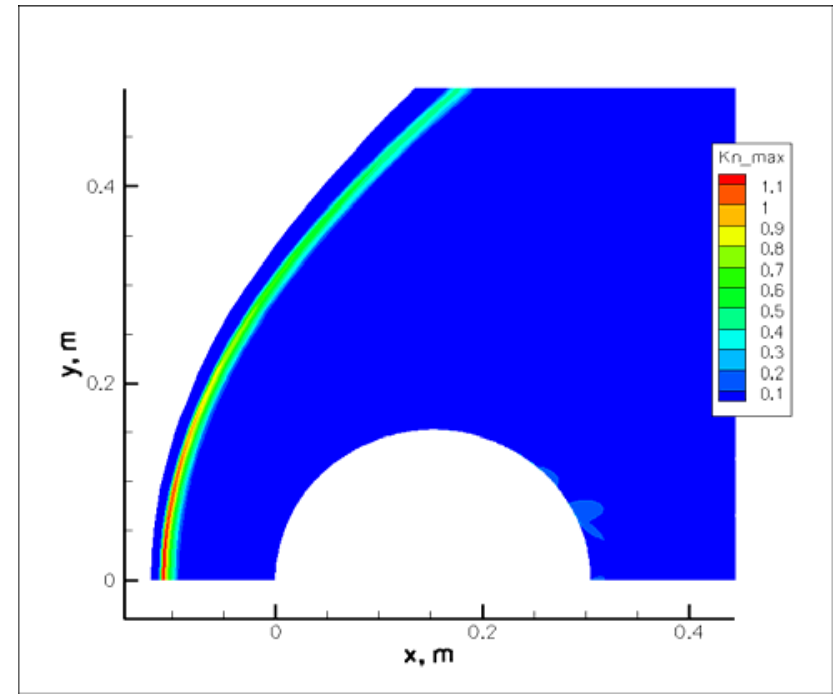

Figure 3. $\mathrm{Kn}=0.01$ maximum gradient length local Knudsen number.

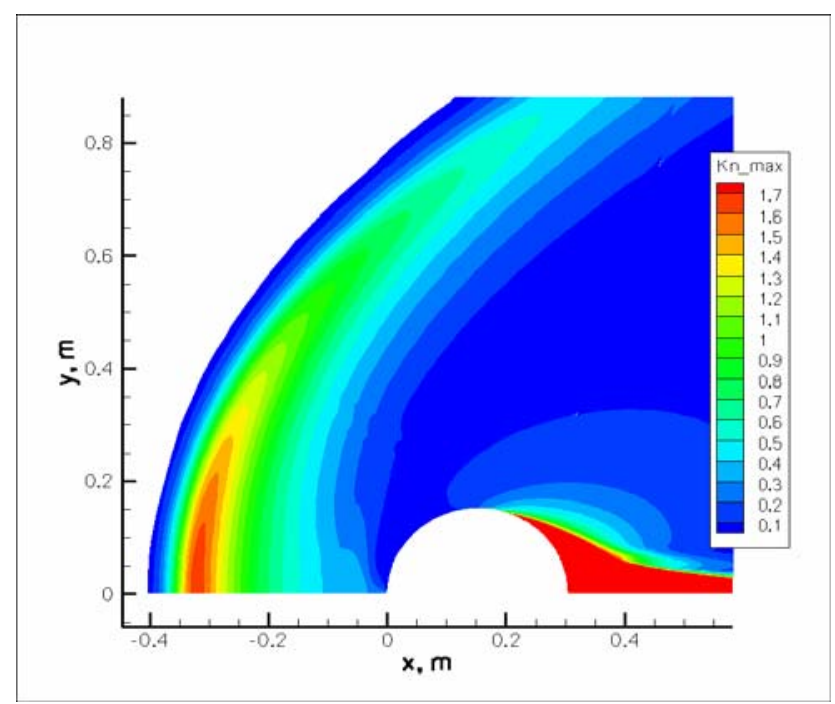

Figure 5. $\mathrm{Kn}=0.25$ maximum gradient length local Knudsen number. 


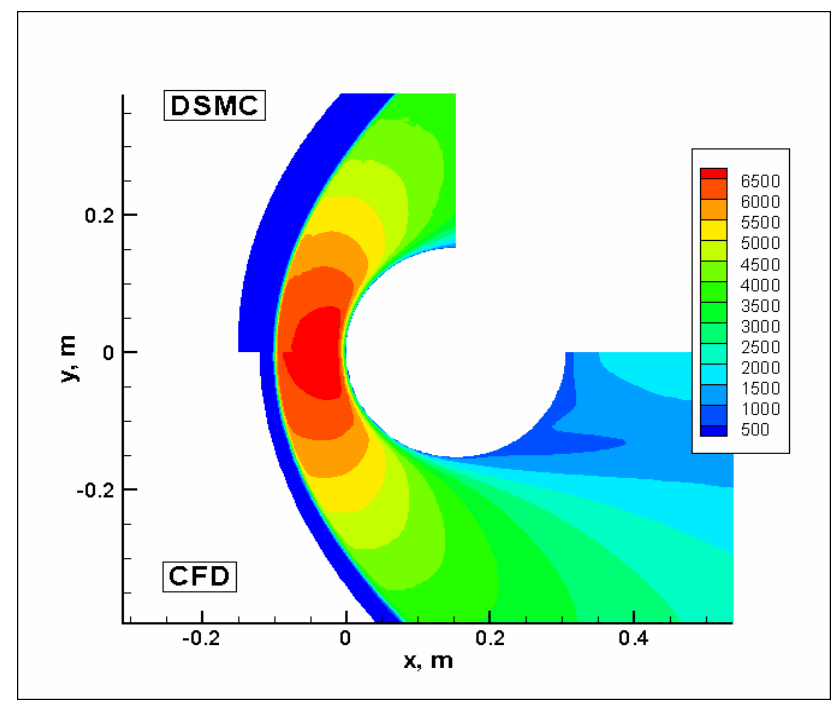

Figure 6. $\mathrm{Kn}=0.002$ temperature $(\mathrm{K})$ field.

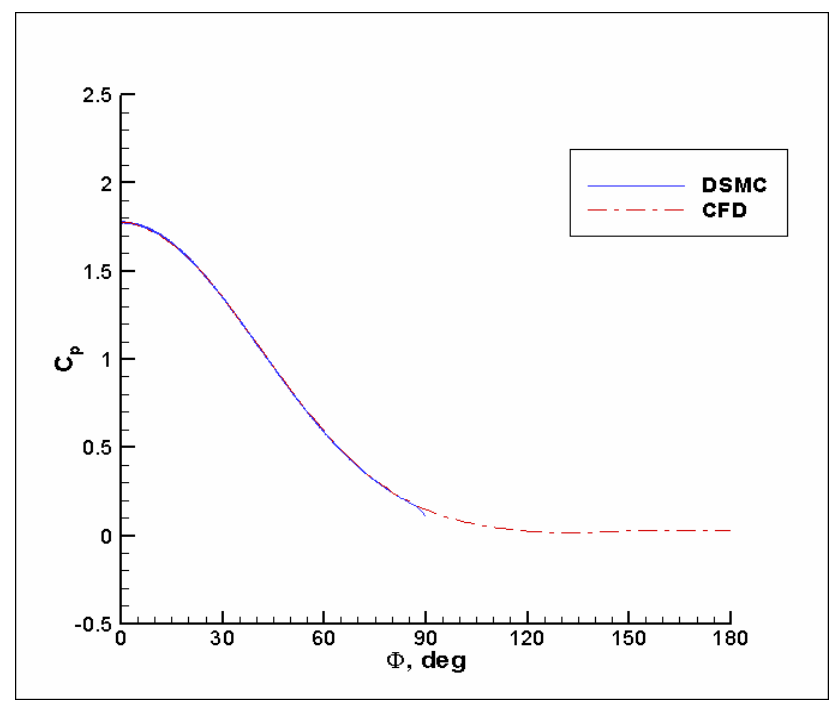

Figure 8. $\mathrm{Kn}=0.002$ surface pressure coefficient.

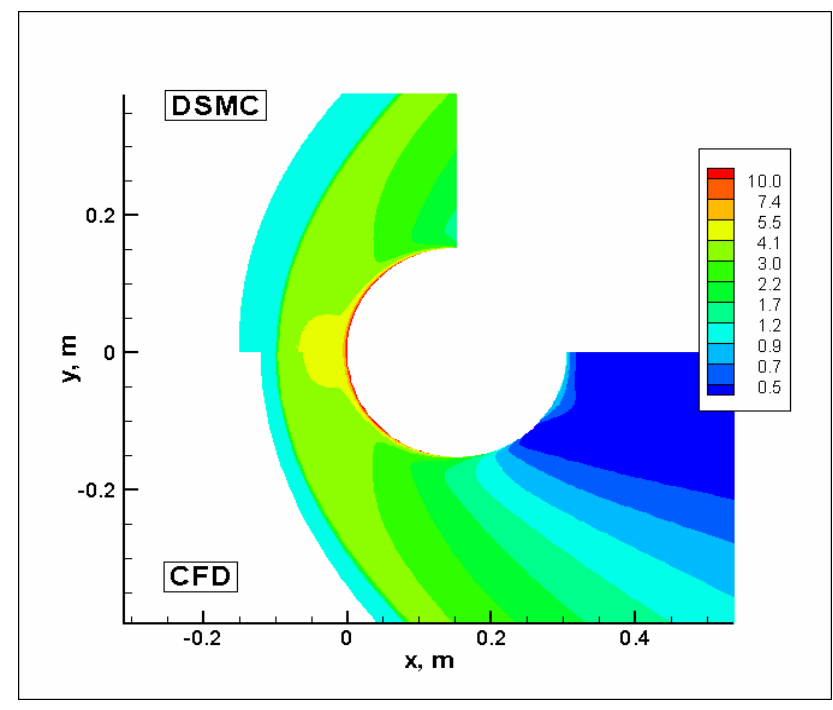

Figure 7. $\mathrm{Kn}=0.002$ density ratio $\left(\rho / \rho_{0}\right)$ field.

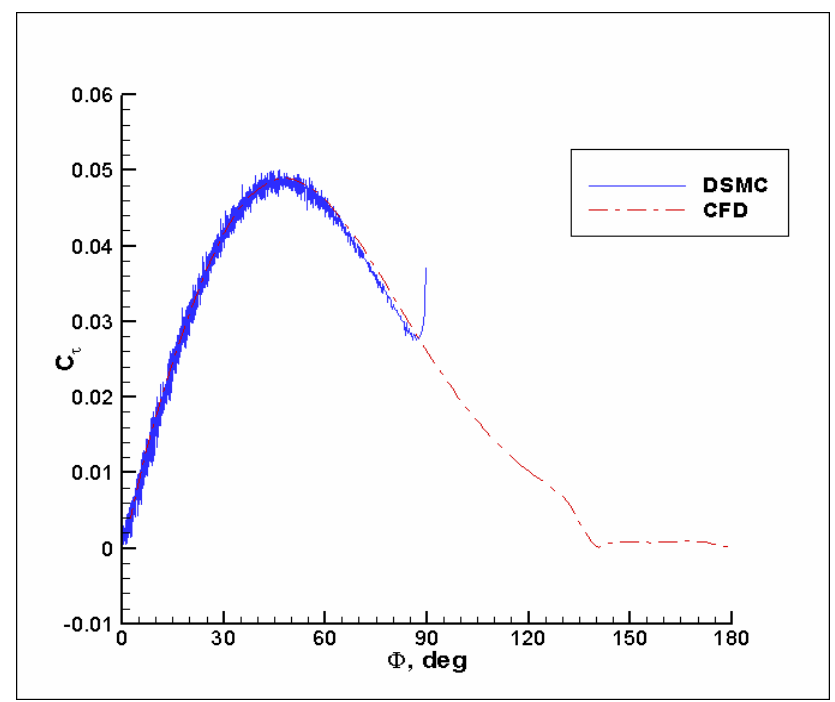

Figure 9. $\mathrm{Kn}=0.002$ surface shear stress coefficient. 


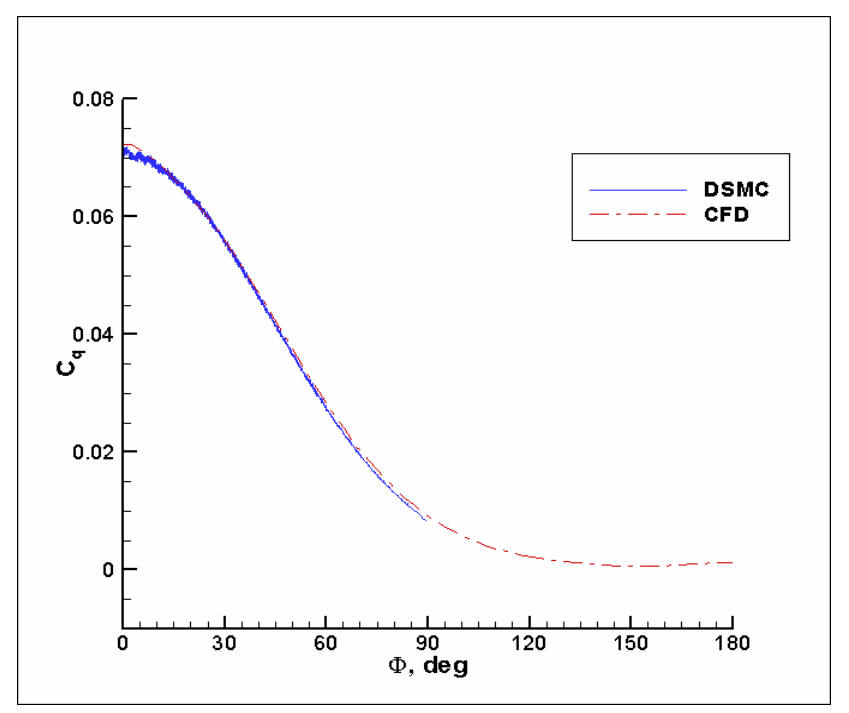

Figure 10. $\mathrm{Kn}=0.002$ surface heat transfer rate coefficient.

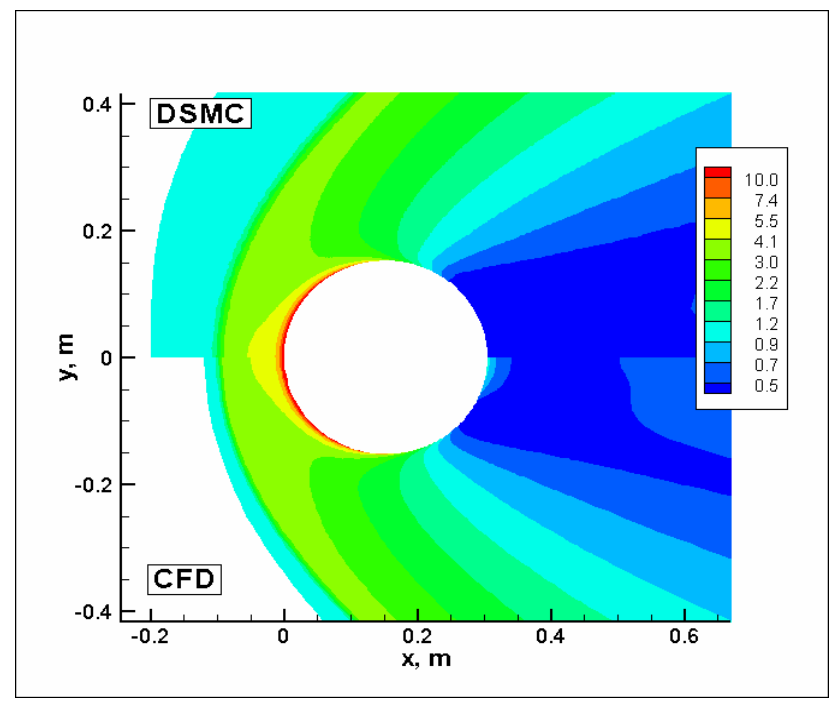

Figure 12. $K n=0.01$ density ratio $\left(\rho / \rho_{0}\right)$ field.

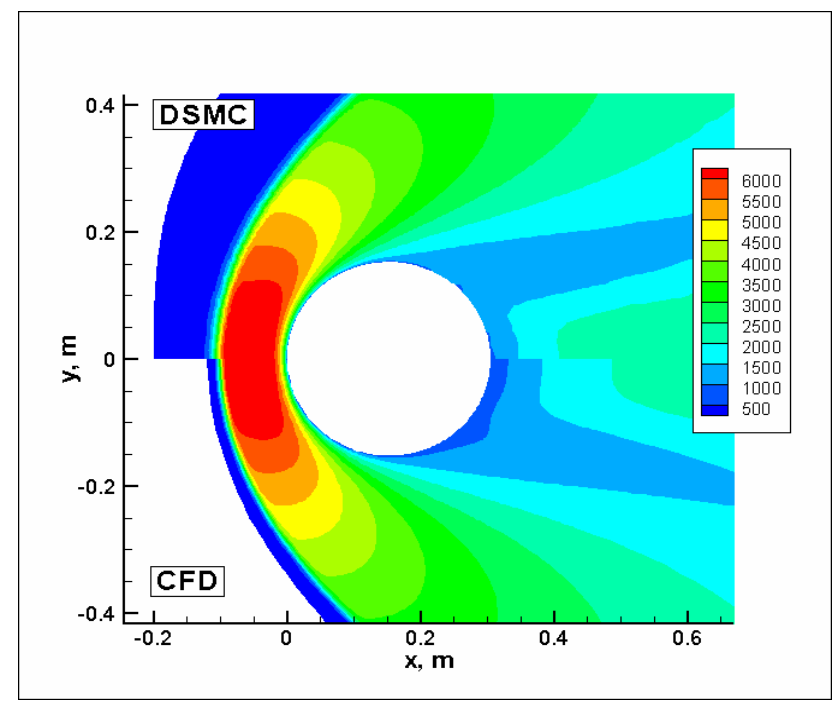

Figure 11. $\mathrm{Kn}=0.01$ temperature $(\mathrm{K})$ field.

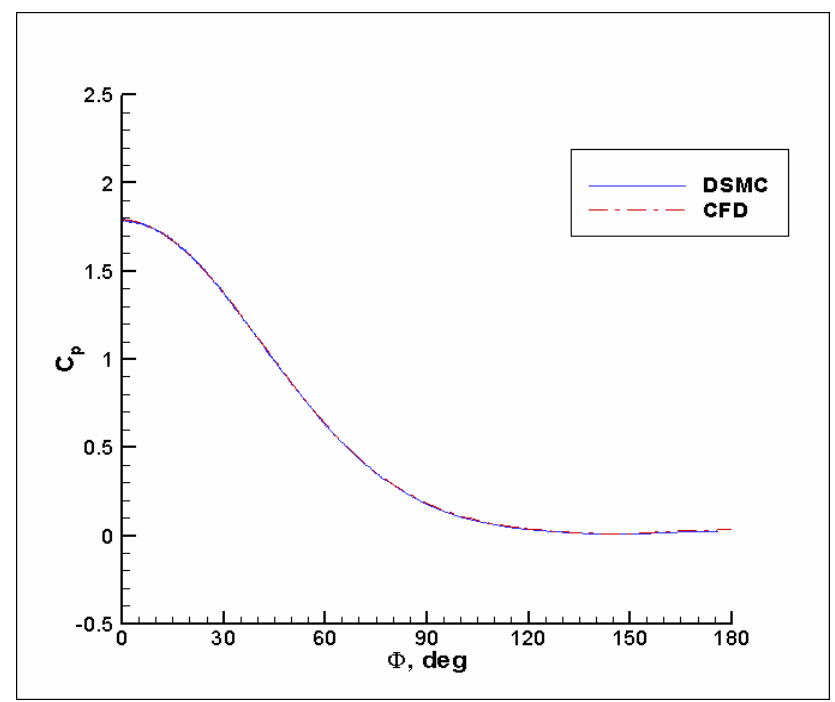

Figure 13. $\mathrm{Kn}=0.01$ surface pressure coefficient. 


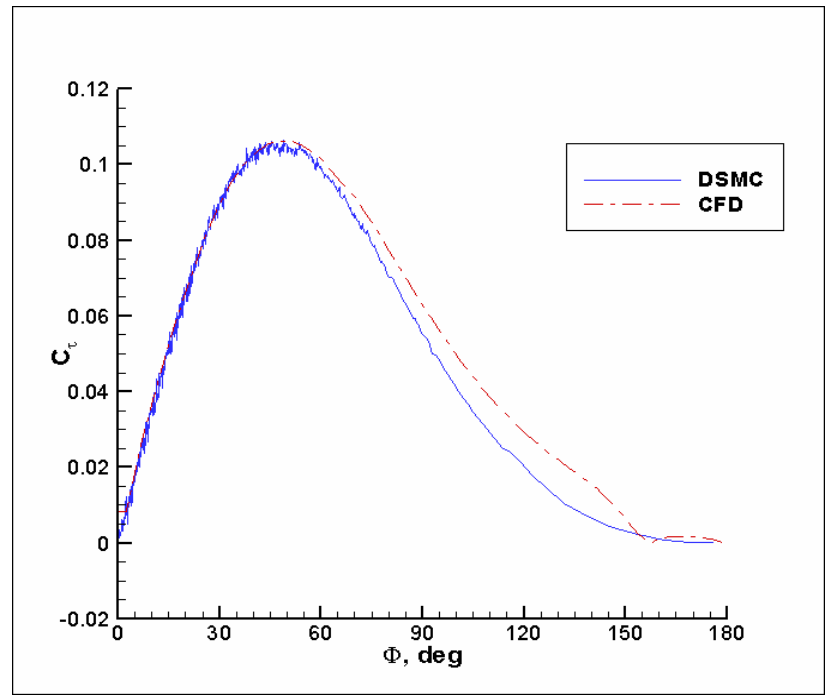

Figure 14. $\mathrm{Kn}=0.01$ surface shear stress coefficient.

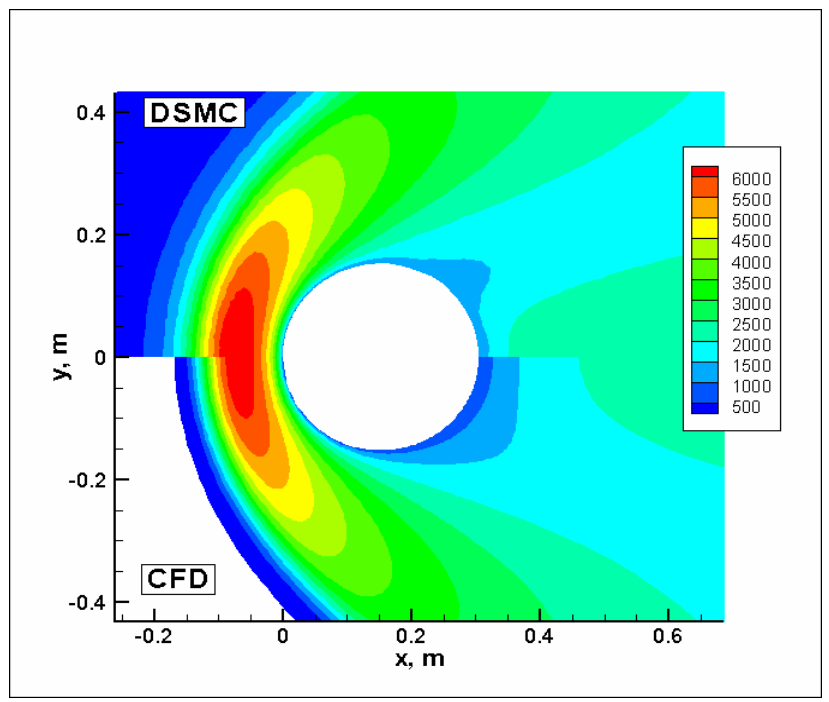

Figure 16. $\mathrm{Kn}=0.05$ temperature $(\mathrm{K})$ field.

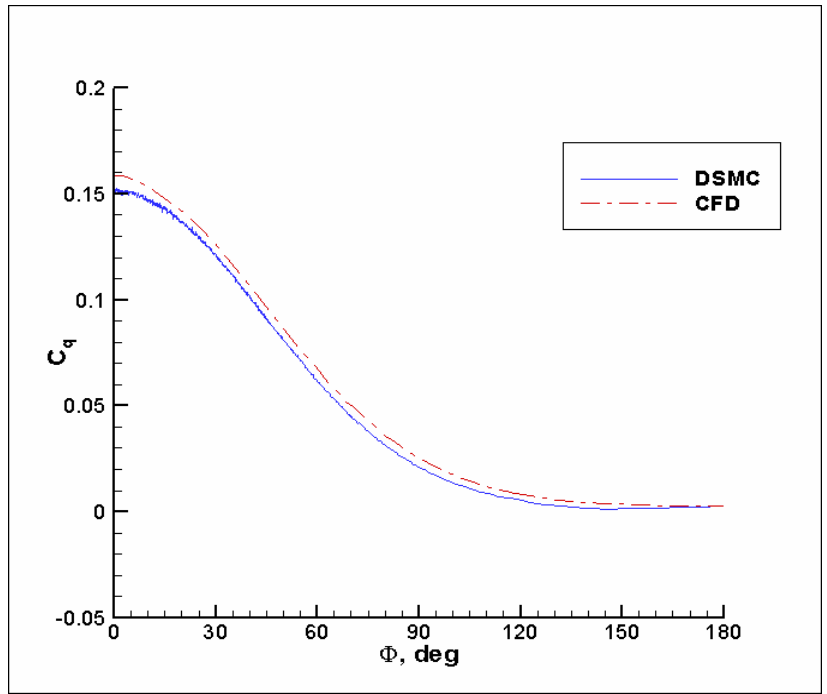

Figure 15. $\mathrm{Kn}=0.01$ surface heat transfer rate coefficient.

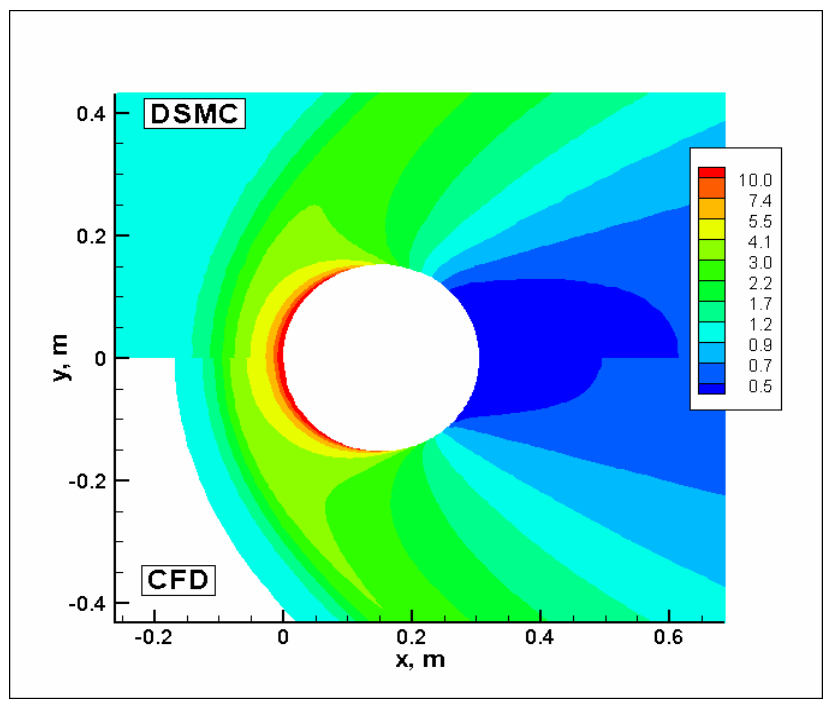

Figure 17. $\mathrm{Kn}=0.05$ density ratio $\left(\rho / \rho_{0}\right)$ field. 


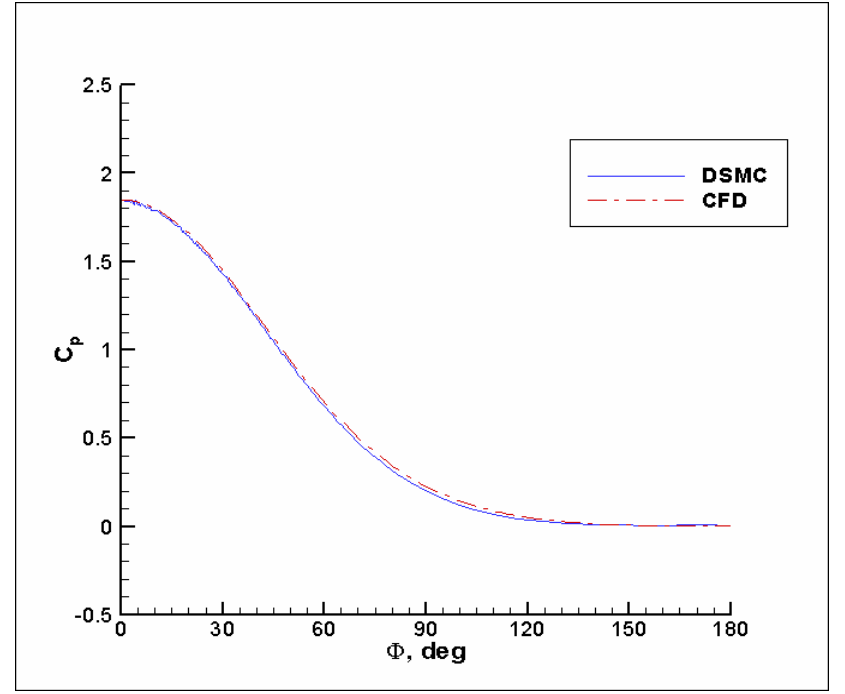

Figure 18. $\mathrm{Kn}=0.05$ surface pressure coefficient.

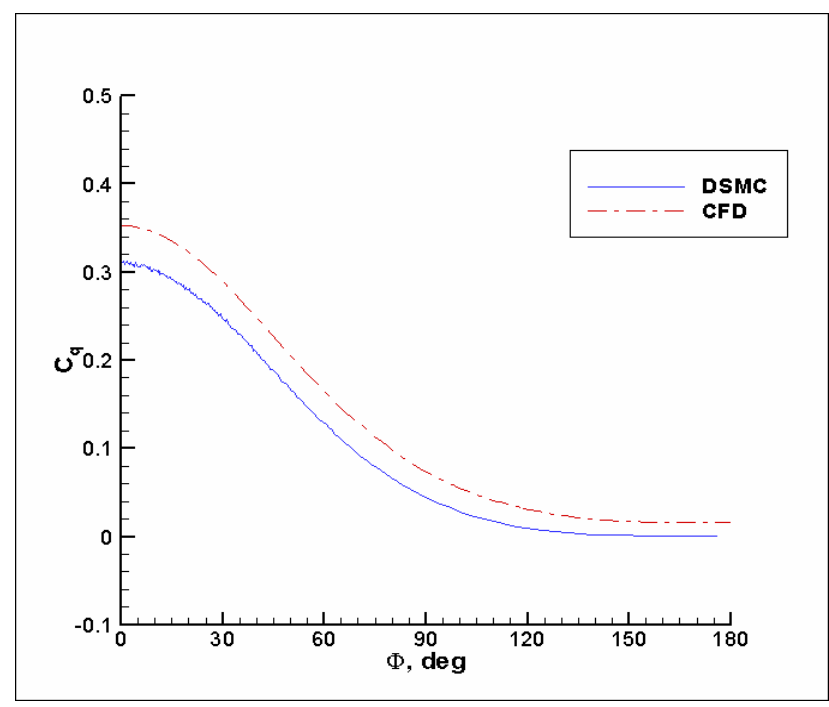

Figure 20. $\mathrm{Kn}=0.05$ surface heat transfer rate coefficient.

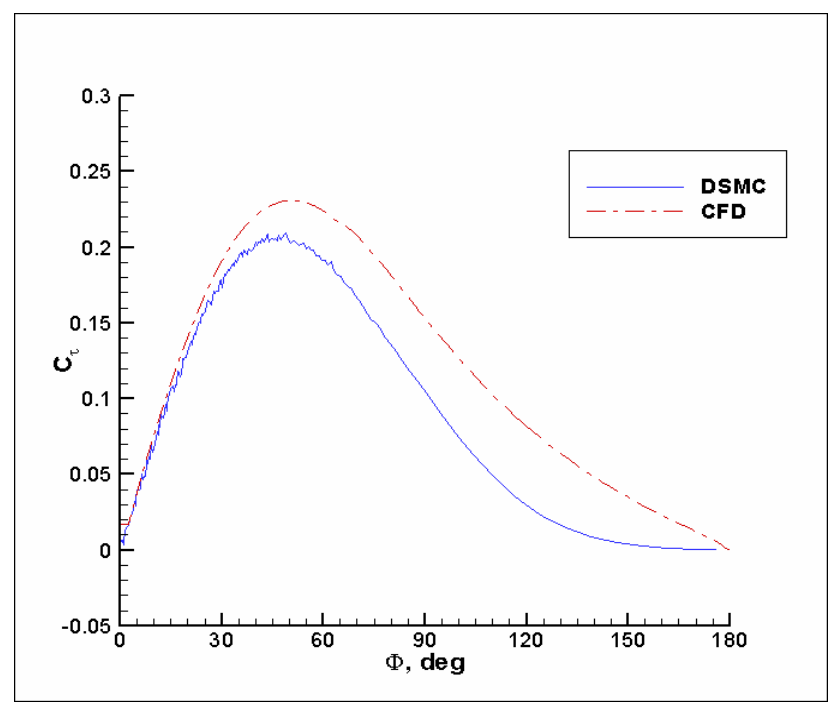

Figure 19. $\mathrm{Kn}=0.05$ surface shear stress coefficient.

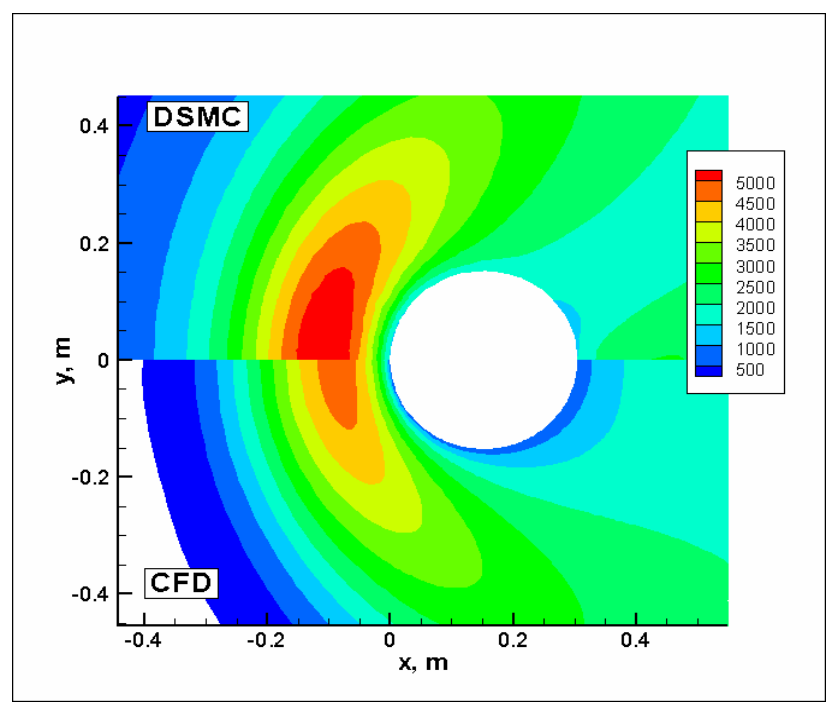

Figure 21. $\mathrm{Kn}=0.25$ temperature $(\mathrm{K})$ field. 


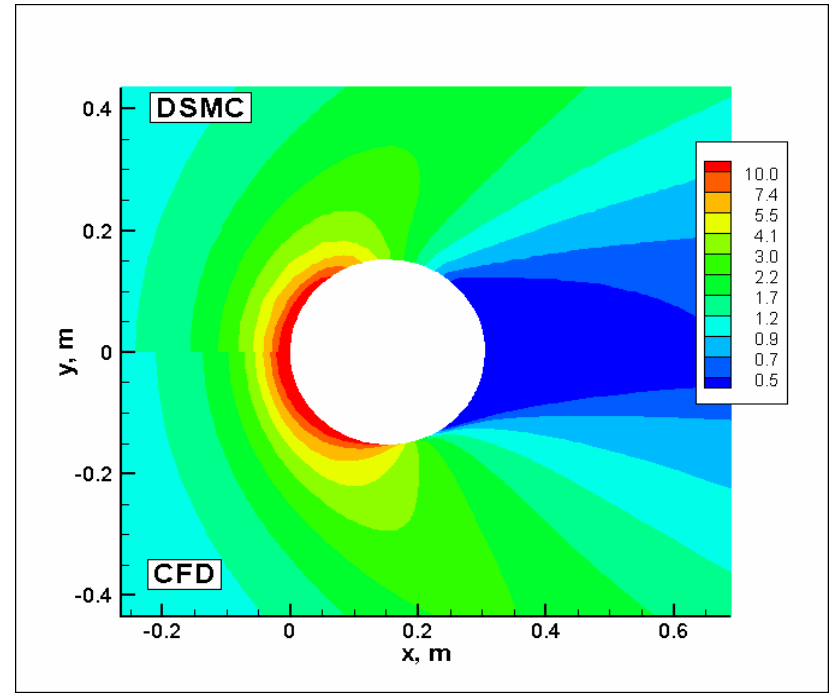

Figure 22. $\mathrm{Kn}=0.25$ density ratio $\left(\rho / \rho_{0}\right)$ field.

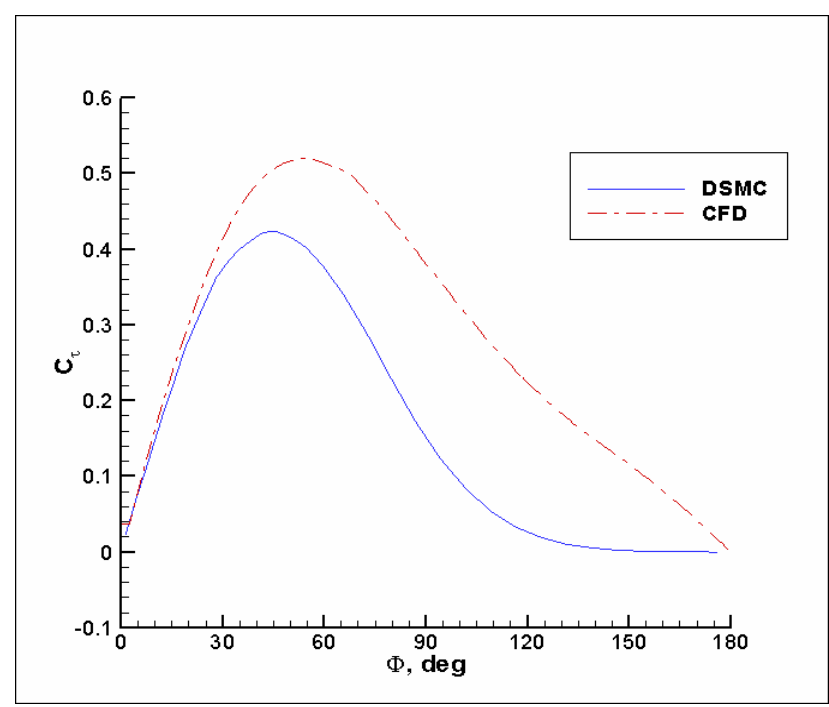

Figure 24. $\mathrm{Kn}=0.25$ surface shear stress coefficient.

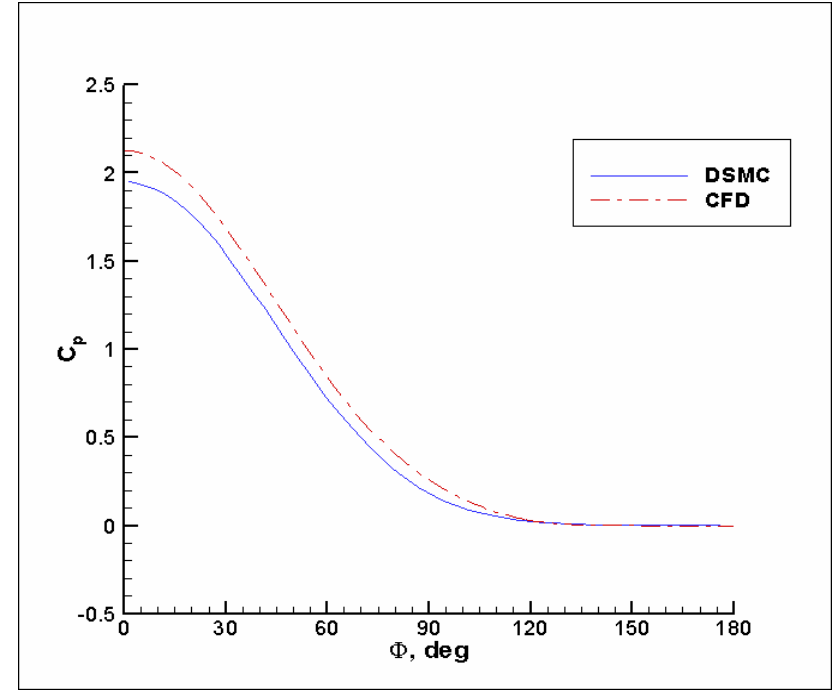

Figure 23. $\mathrm{Kn}=0.25$ surface pressure coefficient.

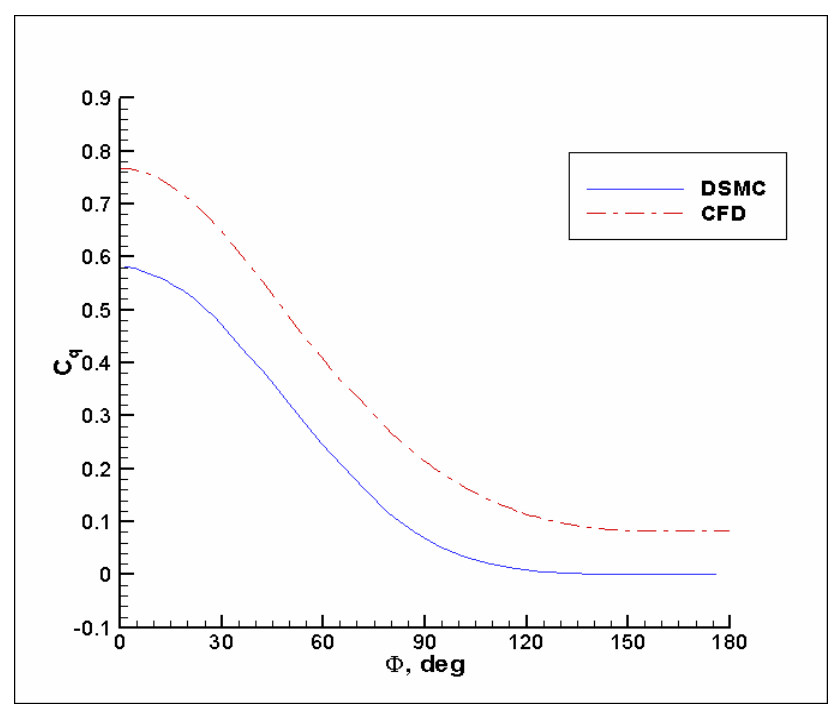

Figure 25. $\mathrm{Kn}=0.25$ surface heat transfer rate coefficient. 


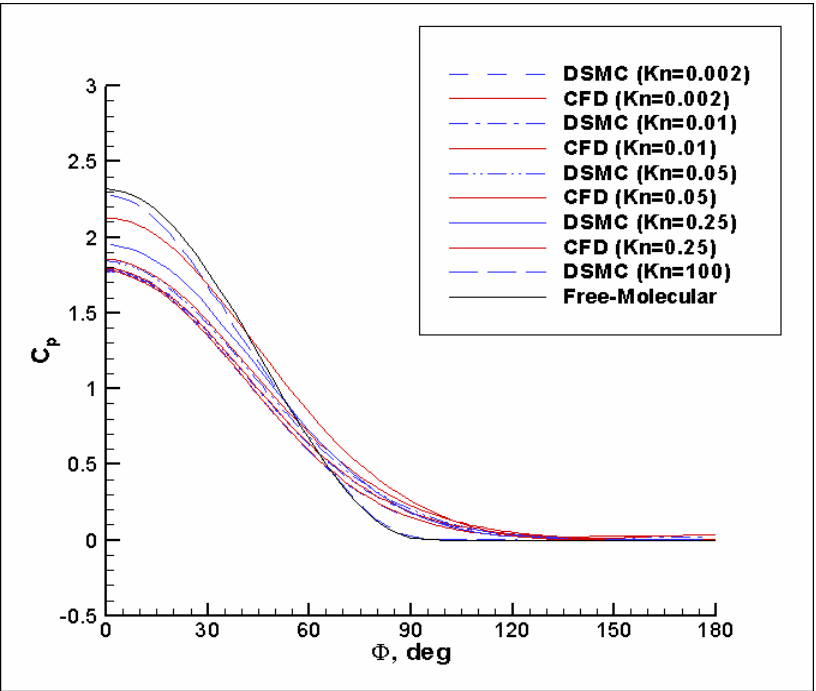

Figure 26. Surface pressure coefficient.

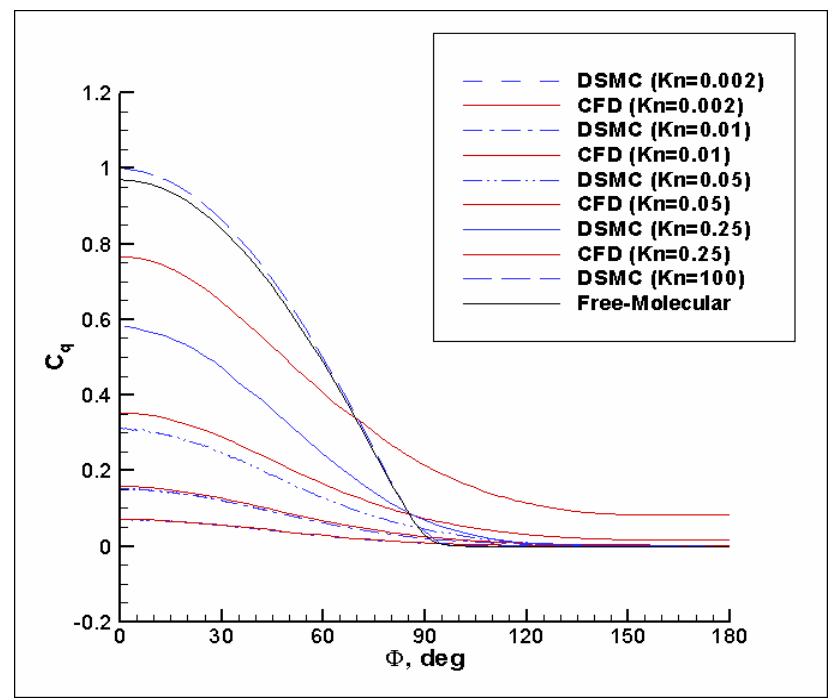

Figure 28. Surface heat transfer rate coefficient.

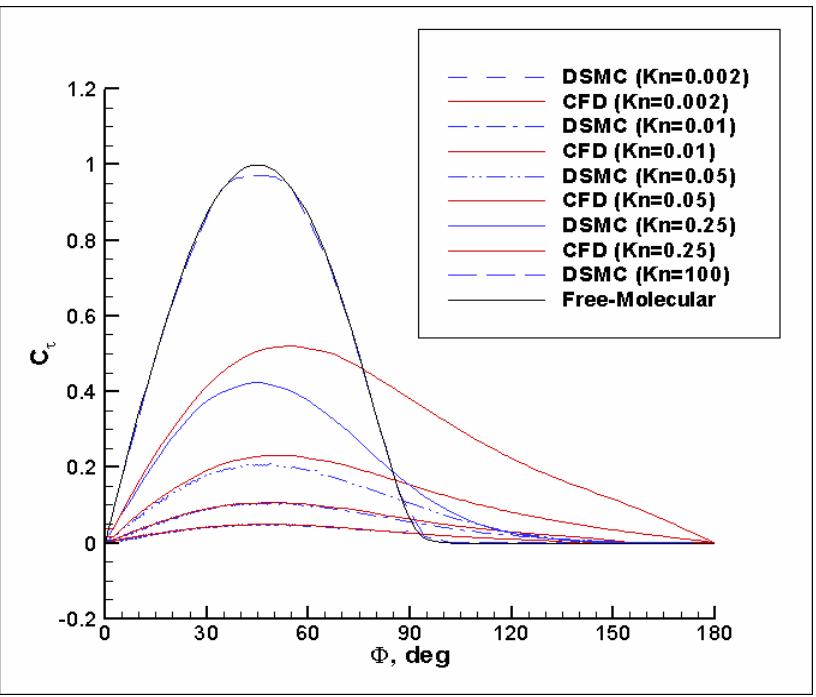

Figure 27. Surface shear stress coefficient. 